\title{
Eops:
}

\section{Duloxetina e incontinencia urinaria de esfuerzo}

\author{
Treatment of stress urinary incontinence with duloxetine
}

Federico Capparelli*

\begin{abstract}
Resumen
La incontinencia urinaria es una condición que se observa en el $35 \%$ de las mujeres adultas, y la incontinencia urinaria de esfuerzo (IUE) es su causa en las tres cuartas partes de los casos.

Este artículo describe el caso de una mujer con incontinencia urinaria de esfuerzo que no responde a la rehabilitación aconsejada. Se formula así la pregunta:¿en mujeres con incontinencia urinaria de esfuerzo la administración de duloxetina disminuye la incontinencia urinaria de esfuerzo?

El autor describe la estrategia de búsqueda bibliográfica y los resultados de la misma, en especial la de dos ensayos clínicos aleatorizados. Se los analiza críticamente y se concluye que a pesar de que la duloxetina reportó resultados beneficiosos, no existe una respuesta contundente a la pregunta. La droga podría tener un efecto beneficioso pequeño en la calidad de vida pero un alto porcentaje de efectos adversos. Se recomienda la estrategia a seguir con la paciente.

Abstract

Urinary incontinence is present in $35 \%$ of adult women, and the stress type is the most frequent presentation.

This article describes the case of a woman with stress urinary incontinence non respondent to rehabilitation maneuvers. The following question arises: Does Duloxetine improves stress urinary incontinence in women?

The author describes the bibliographic research strategy and its results, specially two randomized controlled trials.After their critical analysis there is not a strong answer to the question because Duloxetine appears to have a small benefit in qualiy of life but association with high level of adverse events. We recommend a strategy of patient management.
\end{abstract}

Capparelli F. Duloxetina e incontinencia urinaria de esfuerzo (EOP). Evid Actual Práct Ambul. 2007; 10(5) Set-Oct:157-158

\section{Escenario clínico}

Una mujer de 60 años consulta a su médico clínico de cabecera porque su ginecólogo le ha diagnosticado incontinencia urinaria de esfuerzo. Refiere que le indicaron realizar ejercicios para aumentar el tono del piso pélvico, pero la "cansaron" y no notó mejoría. Ante el fracaso de ese tratamiento el ginecólogo le sugirió un tratamiento quirúrgico 0 un medicamento llamado duloxetina. Los síntomas de incontinencia le generan limitación en la vida diaria y disminución de su calidad de vida, pero no quiere realizar una cirugía. La paciente le pregunta a su médico de cabecera si la alternativa farmacológica le será útil.

\section{Pregunta que generó el caso}

¿En mujeres con incontinencia urinaria de esfuerzo (población) la administración de duloxetina (intervención) disminuye la incontinencia urinaria de esfuerzo (intervención)?

\section{Generalidades}

La incontinencia urinaria es una condición común, crónica y socialmente debilitante, con una prevalencia estimada de $35 \%$ en las mujeres adultas. La incontinencia urinaria de esfuerzo (IUE) es definida por la Sociedad Internacional de Continencia como la queja de salida involuntaria de orina ante el esfuerzo ejercicio o tos. Es la causa más común de incontinencia en mujeres, siendo el $78 \%$ (pura $49 \%$ y mixta $29 \%$ ) de las mujeres con incontinencia.

Actualmente, los ejercicios de entrenamiento de músculos del piso pélvico (EMPP) y las intervenciones conductuales son la terapia de primera línea. Ante la falla la cirugía de continencia es usualmente indicada. La inyección de agentes que abultan la uretra tiene una efectividad a corto plazo y las otras técnicas quirúrgicas, si bien son efectivas en un $90 \%$, no están exentas de riesgos. No existe aún una terapéutica farmacológica indicada en la IUE.

La duloxetina es un inhibidor dual de la recaptación de serotonina y noradrenalina. Su mecanismo propuesto de acción en la mejoría de IUE es la estimulación de descarga del nervio pudendo al esfínter estriado uretral como resultado del aumento de los niveles de serotonina y noradrenalina en los núcleos pudendos en la médula espinal. Así, mientras la duloxetina aumentaría la función del esfínter de músculo estriado vía el nervio pudendo, los músculos del piso pélvico no están inervados por este nervio, sino por eferentes somáticos sacros. De esta forma ambas terapias podrían tener un efecto aditivo en el tratamiento de la IUE.

\section{Estrategia de búsqueda}

Se realizó una búsqueda en MEDLINE con la siguiente estrategia de búsqueda: ("Urinary Incontinence, Stress"[MeSH] AND "duloxetine"[Substance Name]) AND (randomized controlled trial[Publication Type] OR (randomized[Title/Abstract] AND controlled[Title/Abstract] AND trial[Title/Abstract])). Para resolver la pregunta, las siguientes citas fueron seleccionadas de acuerdo a la definición de IUE clínica y urodinámica:

1. Millard RJ, Moore K, Rencken R, Yalcin I, Bump RC; Duloxetine UI Study Group.

Duloxetine vs placebo in the treatment of stress urinary incontinence: a four-continent randomized clinical trial. BJU Int. 2004 Feb;93(3):311-8.

2. Dmochowski RR, Miklos JR, Norton PA, Zinner NR, Yalcin I, Bump RC; Duloxetine Urinary Incontinence Study Group. Duloxetine versus placebo for the treatment of North American women with stress urinary incontinence. J Urol. 2003 Oct; 170(4 Pt 1):1259-63.

3. Ghoniem GM, Van Leeuwen JS, Elser DM, Freeman RM, Zhao YD, Yalcin I, Bump RC; Duloxetine/Pelvic Floor Muscle Training Clinical Trial Group. A randomized controlled trial of duloxetine alone, pelvic floor muscle training alone, combined treatment and no active treatment in women with stress urinary incontinence. J Urol. 2005 May;173(5):1647-53.

Nota: la búsqueda fue realizada a mediados de 2005. una búsqueda a fines de setiembre de 2007 no arroja resultados sustancialmente diferentes (salvo la aparición de un ensayo clínico de tratamiento combinado con rehabilitación, que se excluye en el presente EOP: Filocamo MT y col. Pharmacologic treatment in postprostatectomy stress urinary incontinence. Eur Urol. 2007 Jun; 51(6):1559-64). 


\section{Resumen de la evidencia}

Los primeros dos estudios tienen el mismo diseño:

Diseño: estudio aleatorizado controlado con placebo, doble ciego, de 12 semanas de seguimiento.

Ámbito:

1. Multicéntrico (Argentina, Australia, Brasil, Polonia, Finlandia, España y Sudáfrica).

2. Multicéntrico (Canadá y Estados Unidos).

Población: Se incluyó mujeres $=18$ años con diagnóstico clínico y urodinámico de IUE con $=7$ episodios de incontinencia urinaria (EIU) por semana en los últimos tres meses. Se excluyó pacientes con incontinencia de urgencia.

1. Participaron 558 mujeres de 29 y 79 años, 227 en el grupo intervención y 231 en el grupo placebo. La edad media fue 53 años. Aproximadamente $18 \%$ había recibido tratamiento quirúrgico previo y $12 \%$ realizaba EMPP. Presentaban un promedio de $18 \mathrm{EIU} / \mathrm{semana}$.

2. Participaron 683 mujeres de 22 a 84 años, 344 en el grupo intervención y 339 en el grupo placebo. La edad media fue 53 años. Aproximadamente $13 \%$ había recibido tratamiento quirúrgico previo y $18 \%$ realizaba EMPP. Presentaban un promedio de $18 \mathrm{EIU} / \mathrm{semana}$

\section{Intervención}

Hubo un periodo de 4 semanas de no intervención farmacológica previo a la aleatorización. La intervención fue durante 12 semanas la administración de duloxetina $40 \mathrm{mg}$ cada $12 \mathrm{hs}$. Las pacientes fueron evaluadas en intervalos de 4 semanas durante la fase de tratamiento ciego. Se solicitaba registrar diariamente los EIU en la semana previa a la evaluación.

Medición de resultados principales: Se midió los EIU y un cuestionario validado (puntaje máximo: 100 puntos) de calidad de vida de incontinencia (I-QOL).

El tamaño muestral fue determinado para tener un $80 \%$ de poder para detectar una diferencia de $20 \%$ en el porcentaje de EIU semanales entre placebo e intervención.

Se realizó el análisis según el principio de intención de tratar.

\section{Resultados}

1. Completaron el estudio $92 \%$ de las pacientes de la rama placebo y $75 \%$ de la rama duloxetina (debido a efectos adversos). De las mujeres del grupo intervención, el $60 \%$ tuvo $=50 \%$ de reducción de EIU comparado con el $43 \%$ del grupo placebo $(p<0,001)$. Esto significa una reducción de riesgo absoluto $\left(\mathrm{RRA}^{*}\right)$ de $17 \%$ y un número de pacientes necesario a tratar $\left(\mathrm{NNT}^{\star}\right)$ de 6.

El cambio del I-QOL fue de 10 puntos en el grupo intervención comparado con 6 puntos en el grupo placebo $(p=0,007)$. Hubo un efecto adverso en el $76 \%$ de las pacientes del grupo duloxetina comparado con $59 \%$ del grupo placebo $(p<0,01)$, lo que significa un número de pacientes necesario para dañar (NND*) de 6. Nauseas, ansiedad, mareos e insomnio fueron los efec tos adversos que produjeron mayor discontinuación del ensayo clínico.
2. Completaron el estudio $87 \%$ de las pacientes de la rama placebo y $69 \%$ de la rama duloxetina (debido a efectos adversos). De las mujeres del grupo intervención, el $51 \%$ tuvio $=50 \%$ de reducción de EIU comparado con el $43,2 \%$ del grupo placebo $(p<0,001)$, con un RRA ${ }^{*}$ de $10 \%$ y un $N^{N} T^{*}$ de 10 .

El cambio del I-QOL fue de 11 puntos en el grupo intervención comparado con 7 puntos en el grupo placebo $(p<0,01)$. Hubo un efecto adverso en el $74 \%$ de las pacientes del grupo duloxetina comparado con $50 \%$ del grupo placebo $(p<0,01)$, lo que significa un NND* de 4. Nauseas, ansiedad, mareos e insomnio fueron los efectos adversos que produjeron mayor discontinuación del ensayo clínico

Conclusión: los resultados de ambos estudios muestran mejoría en la continencia urinaria y calidad de vida de las pacientes con IUE. La tasa de efectos adversos menores fue elevada.

\section{Comentarios}

Si bien la diferencia de EIU fue estadísticamente significativa, el beneficio clínico no parece haber sido de magnitud. Por otro lado, ninguno de los dos estudios proveen el intervalo de confianza de 95\% (IC 95\%) de los valores del cambio, con lo cua se deconoce el nivel de precisión de los datos. La diferencia media en escala de calidad de vida (I-QOL) en el grupo intervención comparado con el placebo fue estadísticamente significativa, pero menor a 5 puntos en una escala de 100 puntos, y por ende clínicamente poco relevante. Aproximadamente 25\% de los pacientes abandonaron el tratamiento de intervención farmacológica por efectos adversos, aunque todos los efectos adversos fueron reversibles y no mayores.

Es de destacar el efecto notable observado en el grupo placebo, tanto en el porcentaje de resultados positivos, como en los efectos adversos.

El tratamiento de primera línea para la IUE son los EMPP, con evidencia sostenida en ensayos clínicos aleatorizados de buena calidad. Sin embargo, menos del $20 \%$ de los pacientes enrolados en ambos ensayos estaban recibiendo ese tratamiento y no fue protocolizado en la intervención del estudio. Incluso, los autores postulan en la discusión del segundo estudio que una posibilidad de la menor mejoría respecto a los EIU podría ser el mayor uso de EMPP en la población nórdica. El tercer artículo compara la duloxetina con y sin EMPP contra placebo, pero con un pequeño número de pacientes y con un protocolo de EMPP insuficiente, por lo que no es posible sacar conclusiones robustas.

Finalmente, no existe una respuesta contundente a la pregunta de la paciente. La información disponible sugiere que podría tener un efecto beneficioso de escasa magnitud en la calidad relacionada a la continencia urinaria, pero con un alto porcentaje de efectos adversos. La opción más aconsejable para la paciente de la viñeta sería la realización protocolizada y controlada de EMPP por el especialista, y una vez agotado ese tratamiento, se podría evaluar la incorporación de duloxetina a EMPP para el tratamiento de la IUE, explicando adecuadamente los riesgos y beneficios de esta droga.

\section{Referencias}

1. Millard RJ, Moore K, Rencken R, Yalcin I, Bump RC; Duloxetine UI Study Group. Duloxetine vs placebo in the treatment of stress urinary incontinence: a four-continent randomized clinical trial. BJU Int. 2004 Feb;93(3):311-8

2. Dmochowski RR, Miklos JR, Norton PA, Zinner NR, Yalcin I, Bump RC; Duloxetine Urinary Incontinence Study Group. Duloxetine versus placebo for the treatment of North American women with stress urinary incontinence. J Urol. 2003 Oct;170(4 Pt 1):1259-63.

3. Ghoniem GM, Van Leeuwen JS, Elser DM, Freeman RM, Zhao YD, Yalcin I, Bump RC; Duloxetine/Pelvic Floor Muscle Training Clinical Trial Group. A randomized controlled trial of duloxetine alone, pelvic floor muscle training alone, combined treatment and no active treatment in women with stress urinary incontinence. J Urol. 2005 May;173(5):1647-53. 4. Cardozo L, Drutz HP, Baygani SK, Bump RC. Pharmacological treatment of women awaiting surgery for stress urinary incontinence. Obstet Gynecol. 2004 Sep;104(3):511-9 